\title{
KARL-MARKUS GAUß
}

(IN: GERMANOSLAVICA 31. ZEITSCHRIFT FÜR GERMANO-SLAWISCHE STUDIEN. HILDERSHEIM: GEORG OLMS VERLAG, 2020 (HEFT 1-2). 266 S. ISSN 12109029..)

Mineja Grašič, Philosophische Fakultät, Universität Ljubljana, mineja.grasic@gmail.com

Die einunddreißigste Ausgabe der Fachzeitschrift Germanos/avica erscheint als ein Themenheft über den grenzüberschreitenden österreichischen Schriftsteller Karl-Markus Gauß, der einer breiteren Öffentlichkeit vordergründig durch Das Europäische Alphabet, eine Sammlung kurzer essayistischer Texte zu europäischen Phänomenen in alphabetischer, lexikonartiger Abfolge, bekannt wurde. Die Sekundärliteratur zu diesem vielfältigen Essayisten, Germanisten und Historiker, Chronisten, Feuilletonisten, Publizisten sowie Literatur- und Kulturkritiker ist verhältnismäßig knapp vorhanden (bisher erschienen sind 2007 eine Monographie von Christian Tanzer bei Dieter Heinz Verlag sowie zwei Sammelbände, 2010 bei Zsolnay Verlag Grenzgänge: der Schriftsteller Karl-Markus Gauß (hrsg. von Herbert Ohrlinger und Daniela Strigl) sowie 2017 bei Otto Müller Verlag Von der Produktivkraft des Eigensinns. Die Literaturen des Karl-Markus Gauß (hrsg. von Werner Michler, Klemens Renoldner und Norbert Christian Wolf), daher ist das Themenheft der Germanoslavica, das neben Siegfried Ulbrecht noch Edgar Platen und Irena Samide als Gastherausgeber betreut haben, eine willkommene Perspektiverweiterung. Das Heft geht auf eine dreitägige Tagung mit dem Titel Europa-Mitteleuropa-Österreich. Peripherien und Minoritäten bei Karl Markus Gauß zurück, das vom 24. bis zum 26. Oktober 2018 in Ljubljana stattgefunden hat und von den Vertretern der Universitäten Göteborg (Institut für Sprachen und Literaturen/Forum für deutsche Gegenwartsliteratur und Deutschlandstudien) und Ljubljana (Abteilung für Germanistik mit Niederlandistik und Skandinavistik) in Zusammenarbeit mit dem Slawischen Institut der Akademie der Wissenschaften der Tschechischen Republik (Prag) organisiert wurde.

Karl-Markus Gauß wurde 1954 in Salzburg geboren. Nach dem Studium der Germanistik und Geschichte fing er schon früh an, sich als 
Autor und Publizist zu betätigen, seit 1991 gibt er u. a. die Literaturzeitschrift Literatur und Kritik heraus. Heute gilt er als einer der wichtigsten Vertreter der Europaessayistik, wobei sein Fokus nicht nur auf den Rändern Europas liegt, sondern vor allem auf europainternen Grenzen und Rändern, die sich historisch verschoben haben und sich gegenwärtig noch immer verschieben. Gauß schreibt für diverse Tageszeitungen wie Süddeutsche Zeitung, Neue Zürcher Zeitung, Die Presse u.v.a. Es wurden ihm zahlreiche Preise und Auszeichnungen verliehen, darunter auch der Europäische Essaypreis Charles Veillon, der Johann-Heinrich-Merck-Preis oder der Jean-Améry-Preis. In seinen Werken beschreibt Gauß Menschen, die ins Randständige, in die Periphere, in Armut und ins Vergessen gedrängt sind.

Das Themenheft wird mit dem Einleitungsbeitrag Von Europas Mittelpunkt(en). Zur ,literarischen Landkarte' von Karl-Markus Gauß' Essayistik eröffnet, in dem der Herausgeber Edgar Platen, Professor für Germanistische Literaturwissenschaft an der Universität Göteborg versucht, mit Hilfe von Gauß' essayistischem Schreiben eine ,literarische Landkarte' Europas zu erstellen und in dieser einen Mittelpunkt zu bestimmen. Platen orientiert sich dabei am Eisernen Vorhang und an der Donau, berücksichtigt aber keine anderen geopolitischen Faktoren, sondern schöpft vielmehr an Gauß' kulturologischen Interessen.

Im darauffolgenden Beitrag, verfasst von den Literaturwissenschaftlern Helena Ulbrechtová und Siegfried Ulbrecht, werden die literarischen Welten von Karl-Markus Gauß und Martin Pollack mit Hilfe der vergleichenden Methode analysiert, wobei die Autoren des Beitrags Unterschiede und Parallelen zwischen den beiden Europabildern suchen. Im Zentrum der Studie liegt die räumlichsemantische Analyse der Region der Gottschee.

Die Autorinnen der dritten Abhandlung mit dem Titel Die (un)bekannten Gottscheer: Von der gedruckten Geschichte zur virtuellen Heimat sind Irena Samide und Tanja Žigon, Professorinnen an der Philosophischen Fakultät in Ljubljana. Der Beitrag behandelt die Gottschee und die Gottscheer, die im 14. Jahrhundert dorthin gezogen waren und im Dritten Reich ihre Heimat verlassen mussten. Hier werden drei Aspekte der Gottscheer-Vergangenheit dargestellt: Während zunächst die langjährige journalistische Aktivität in der GottscheeRegion beleuchtet und danach dem Schicksal der Gottscheer nachgegangen wird, wie es sich in dem Gaußschen Essay Der Wald der Geschichte aus seinem Band Die sterbenden Europäer abzeichnet, fokussiert sich der dritte Teil auf die Suche nach persönlichen Spuren der Gottscheer Vergangenheit, die sich immer mehr in das Virtuelle verlegt. 
Frank Thomas Grub vom Institut für moderne Sprachen an der Uppsala Universität in Schweden beschäftigt sich mit Gauß' Werken, die der Autor selbst als, Journals' bzw. ,Jahresbuch' klassifiziert, und zwar mit Mit mir, ohne mich (2002) und Von nah, von fern (2003). Bei seiner Analyse liegt der Fokus auf thematischen Kontinuitäten, darunter historischen und zeitgenössischen Peripherien Europas, ihrer Population und Identitätsfragen.

Germanistin Stephanie Bettina Schwarzendorfer untersucht die Bedeutung vierer bestimmter Wörter in Gauß' Buch Abenteuerliche Reise durch mein Zimmer, wobei sie auf Gauß' Verstehen und Kritizismus Europas Hinsicht nimmt. Die Forscherin findet in diesen Wörtern Bibliothek, Bücher, Duschhauben und Tassen - Metaphern für ein hybrides Europa, Gauß' Persönlichkeit und sein immer intensiveres Interesse an Minderheiten.

Der nächste Beitrag, betitelt Am Rand der Ränder von Karl-Markus Gau $\beta^{\prime}$ Schreiben: Italien, stammt von Iginia Barretta, Doktorandin an der Universität Göteborg, und fokussiert sich auf die Frage, wieso Italien auf Gauß' literarischer Europakarte oft in Verbindung mit Südosteuropa erscheint, obwohl es tatsächlich nicht dazu gehört. Zugleich behandelt diese Studie die Problematik von Gauß' doppeldeutiger, komplexer und unerwarteter Italiendarstellung.

Im Beitrag Der lange Nachkrieg in Das Erste, was ich sah von KarlMarkus Gauß setzt sich Johann Georg Lughofer von der Philosophischen Fakultät in Ljubljana mit Gauß' autobiographischem Buch auseinander. Es werden explizite sowie implizite Folgen von Nationalsozialismus in der Nachkriegsperiode in Salzburg der späten 50er Jahre analysiert, darunter pädagogische Erziehung, Spiele, Namenwahl und der Opfermythos.

Mit Gauß' autobiographischem Buch Das erste, was ich sah beschäftigt sich auch der Germanist Václav Maidl, der dieses in Verbindung mit Friedrich Brandls Büchern Ziegelgassler und Glock'n'Roll analysiert. Alle drei Texte sprechen von der Kindheit in der Nachkriegszeit. Maidl zieht Parallelen zwischen Memoiren der beiden Buchautoren und seinen eigenen Erinnerungen, wobei er trotz geographischer und politischer Unterschiede zahlreiche Gemeinsamkeiten findet.

Da sowohl Karl-Markus Gauß als Ilma Rakusa 2005 der VilenicaLiteraturpreis verliehen wurde, schreibt Vesna Kondrič Horvat von der Philosophischen Fakultät in Maribor (Slowenien) in ihrem Beitrag Wo ist die Weltmitte? über die literarischen Erfolge beider Autoren. Sie hebt den Wert dieser zwei Autoren und ihrer Werke sowie ihren Einfluss auf die Nachwelt hervor.

Der nächste Beitrag, den die Translationswissenschaftlerin Tanja Žigon, die sich in diesem Sammelband bereits zum zweiten Mal vorstellt, 
und die Germanistin an der Universität in Ljubljana Petra Kramberger verfassten, setzt sich mit der Rezeption von Karl-Markus Gauß im slowenischen Raum auseinander. Die Studie stellt Übersetzungen von Gauß' Werken ins Slowenische vor und präsentiert Gauß' reproduktive und passive Rezeption im slowenischen Raum sowie sein Profil in slowenischer Literatur und Kultur.

Die slowenische Germanistin und Literaturwissenschaftlerin Mira Miladinović Zalaznik, Übersetzerin von Gauß’ Werken, reflektiert in ihrem Beitrag ihre übersetzerische Arbeit an drei Texten (Das Europäische Alphabet, Die sterbenden Europäer und Die Hundeesser von Svinia). Dabei sucht die Autorin ihre eigenen Annotationen im sozio-historischen Kontext und beschreibt ihre Begegnung mit Karl-Markus Gauß im - für Slowenien und Gauß selbst - äußerst bedeutenden Jahr 1991.

Der Artikel der Professorin Milka Car Prijić aus Zagreb setzt sich zum Ziel, das Europabild in nicht-fiktionalen Werken von Karl-Markus Gauß und Miroslav Krleža zu analysieren und dabei die Rolle des Übersetzers als kulturellen Mediators hervorzuheben. Im Fokus des Beitrags steht Karl Markus Gauß als jemand, der eine bedeutende Rolle in der Rezeption des kroatischen Autors Miroslav Krleža in den deutschsprachigen Gebieten spielt.

Der österreichische Schriftsteller und Germanist Christoph Janacs ist Verfasser vom dreizehnten und letzten Beitrag dieses Themenhefts, in dem er in die Fußspuren von Karl-Markus Gauß tritt, um mit dem Autor eine fiktive Konversation über seine Arbeitsmethoden, Interessen, Reisen und die Themen seines Schreibens zu führen. Durch eine Kombination von Beschreibung, Beobachtung und Besprechung gelingt es Janacs, ein emphatisches Bild von Gauß zu erstellen.

Diese Ausgabe der Germanos/avica gibt einen differenzierten und aufschlussreichen Einblick in das Schreiben des österreichischen Autors Karl-Markus Gauß. Die oben besprochenen Beiträge von fünfzehn Expertinnen und Experten aus verschiedenen Fachgebieten zeichnen sich durch theoretische Fundiertheit und wissenschaftliche Exaktheit aus und bringen den Leser einen Schritt näher an Karl-Markus Gauß und seine Essayistik. Hervorzuheben sind vor allem die Bandbreite der Beiträge, die interdisziplinäre Herangehensweise sowie die interkulturelle Sicht, die viele, bisher noch nicht erforschte kulturelle, historische, translatorische und literarische Wechselbeziehungen in den Vordergrund stellt. Daher kann angenommen werden, dass das Themenheft reges Interesse nicht nur bei den Literaturwissenschaftlern, sondern bei einer breiten internationalen Leserschaft finden wird. 